\title{
Power-Law Spin Correlations in a Perturbed Spin Model on a Honeycomb Lattice
}

\author{
K. S. Tikhonov, ${ }^{1,2}$ M. V. Feigel'man, ${ }^{1,2}$ and A. Yu. Kitaev ${ }^{3}$ \\ ${ }^{1}$ L. D. Landau Institute for Theoretical Physics, Kosygin street 2, Moscow 119334, Russia \\ ${ }^{2}$ Moscow Institute of Physics and Technology, Moscow 141700, Russia \\ ${ }^{3}$ California Institute of Technology, Pasadena, California 91125, USA
}

(Received 12 September 2010; revised manuscript received 3 January 2011; published 11 February 2011)

We consider the spin- $\frac{1}{2}$ model on the honeycomb lattice in the presence of a weak magnetic field $h_{\alpha} \ll 1$. Such a perturbation destroys the exact integrability of the model in terms of gapless fermions and static $Z_{2}$ fluxes. We show that it results in the appearance of a long-range tail in the irreducible dynamic spin correlation function: $\left\langle\left\langle s^{z}(t, r) s^{z}(0,0)\right\rangle\right\rangle \propto h_{z}^{2} f(t, r)$, where $f(t, r) \propto[\max (t, r)]^{-4}$ is proportional to the density polarization function of fermions.

DOI: 10.1103/PhysRevLett.106.067203

PACS numbers: $75.10 . J m$

Quantum spin liquids (QSLs) (see, e.g., Refs. [1-6]) present examples of strongly correlated quantum phases which do not develop any kind of local order, while their specific entropy vanishes at zero temperature. Critical, or algebraic, QSLs are characterized by spin correlation functions that decay as some power of distance and time. In some cases, the correlation asymptotics can be deduced from a representation of spin operators in terms of almostfree fermions [7]. However, a complete calculation based on a microscopic Hamiltonian has not been demonstrated due to the lack of suitable exactly solvable models (in more than one spatial dimension).

We show in this Letter that the anisotropic spin- $\frac{1}{2}$ model on the honeycomb lattice, proposed by one of us [8], can be used as a starting point for the construction of an analytically treatable critical QSL. This result may seem surprising since it is known [9] that the original model [8] possesses no spin correlations at distances longer than a single lattice bond. We will see, however, that a small perturbation of the model [8], e.g., a weak external magnetic field, is sufficient to "turn on" long-range spin correlations, albeit with a small overall prefactor. Thus we disagree with the statement made in Ref. [9] that the shortrange character of spin correlations survives in the presence of a weak magnetic field. Very recently, a weakly perturbed model of Ref. [8] was studied numerically in [10] in relation with experiments [11]. We consider the model defined by the Hamiltonian

$$
\mathcal{H}=J \sum_{l=\langle i j\rangle}\left(\sigma_{i} \mathbf{n}_{l}\right)\left(\sigma_{j} \mathbf{n}_{l}\right)-\sum_{i} \mathbf{h}_{i} \sigma_{i}
$$

Unit vectors $\mathbf{n}_{l}$ are parallel to the $x, y$, and $z$ axes for the corresponding links $x, y$, and $z$ of the honeycomb lattice; see Fig. 1. At $\mathbf{h}_{i} \equiv 0$ the Hamiltonian (1) was solved exactly [8] via a mapping to a free fermion Hamiltonian. In this approach, each spin $\sigma_{i}$ is represented in terms of four Majorana operators $c_{i}, c_{i}^{x}, c_{i}^{y}, c_{i}^{z}$ with the following anticommutation relations: $\left\{c_{i}^{\alpha}, c_{j}^{\beta}\right\}=2 \delta_{i j} \delta_{\alpha \beta}$, so that $\sigma_{i}^{\alpha}=i c_{i} c_{i}^{\alpha}$. In terms of these new operators, the zero-field Hamiltonian reads $\mathcal{H}=-i J \sum_{\langle i j\rangle} c_{i} u_{i j} c_{j}$, and $u_{i j}=i c_{i}^{\alpha} c_{j}^{\alpha}$ are constants of motion: $\left[\mathcal{H}, u_{i j}\right]=0$, with $u_{i j}= \pm 1$. The ground state $|G\rangle$ corresponds to an arbitrary choice of $\left\{u_{i j}\right\}$ that minimizes the energy. It is convenient to introduce the notion of $Z_{2}$ flux, defined for each hexagon $\pi$ as a product $\phi_{\pi}=\prod u_{i j}$ (since $u_{i j}=-u_{j i}$, we have to choose a particular ordering in this definition: $i \in$ even sublattice, $j \in$ odd sublattice). The ground state of this model is a symmetrized sum of states with different sets of integrals of motion $\left\{u_{i j}\right\}$, corresponding to all fluxes equal to 1 . For practical calculations of physical quantities, one does not have to implement such symmetrization and can assume that all $u_{i j} \equiv 1$. We denote by $H$ the corresponding Majorana Hamiltonian: $H=-i J \sum_{\langle i j\rangle} c_{i} c_{j}$. It can be diagonalized with the use of Fourier transformation. The spectrum of the resulting free fermions is gapless and has two conic points. To begin, we recall the calculation of the spin-spin correlation function $g_{i j}^{\alpha \beta}=\left\langle\sigma_{i}^{\alpha}(t) \sigma_{j}^{\beta}(0)\right\rangle$ in the unperturbed model with $h \equiv 0$ [9]. Spin operator $\sigma_{i}^{\alpha}$ acting

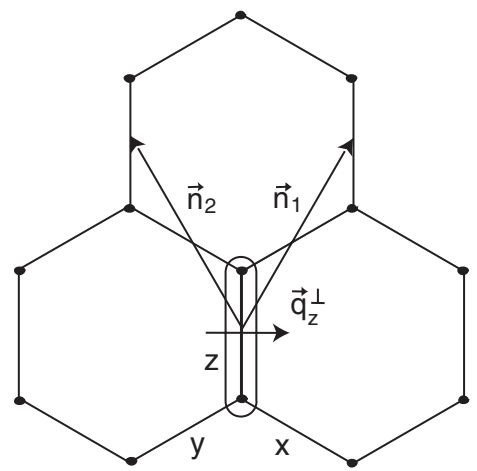

FIG. 1. A honeycomb lattice fragment with the $z-z$ link that belongs to a given elementary cell. The indicated vector $\mathbf{q}_{z}^{\perp}=\hat{x}$ shows the direction of oscillations found in the $\left\langle s^{z}(0) s^{z}(\mathbf{r})\right\rangle$ correlation function. 
on the ground state produces two $Z_{2}$ fluxes and creates a fermion. Since states with different flux configurations are mutually orthogonal and fluxes do not move in the process of time evolution governed by the Hamiltonian $\mathcal{H}$, a nonzero result for the correlation function is possible only if the second spin operator $\sigma_{j}^{\beta}$ creates the same pair of fluxes. Thus the sites $i$ and $j$ are either the same or nearest neighbors. For larger separations between $i$ and $j$, one has $g_{i j}=0$. However, this result is due to the static nature of $Z_{2}$ fluxes; furthermore, the ground state is a linear combination of states with the same flux pattern. Both these features are destroyed by any perturbation (for example, external magnetic field) that does not commute with operators $\left\{u_{i j}\right\}$.

In this Letter, we consider the honeycomb lattice model with an external magnetic field, which is treated as a weak perturbation. Before delving into calculations, we note that a generic magnetic field opens a gap $\Delta$ in the fermionic spectrum [8], with $\Delta \sim h_{x} h_{y} h_{z} / J^{2}$. In what follows we neglect this gap. This is definitely possible if one of the field components vanishes, i.e., if the field is directed in one of the coordinate planes. For the generic field direction, our results for spin correlations are applicable for intermediate distances, $1 \ll r \ll 1 / \Delta$. For simplicity we discuss the total spin in the $r$ th elementary cell, $s_{r}=$ $\sigma_{r, 1}+\sigma_{r, 2}$, and calculate $z-z$ correlations only. Since the external magnetic field induces finite magnetization, $\left\langle s_{r}^{z}\right\rangle \neq 0$, we study the irreducible correlation function: $g(t, \mathbf{r})=\left\langle\left\langle s_{r}^{z}(t) s_{0}^{z}(0)\right\rangle\right\rangle$. We are interested in the long-time and/or long-distance asymptotics of $g(t, \mathbf{r})$.

It is convenient to introduce complex bond fermions, defined as follows: $\psi_{r}=\frac{1}{2}\left(c_{r, 1}+i c_{r, 2}\right)$ and $\phi_{r}=\frac{1}{2}\left(c_{r, 1}^{z}+\right.$ $\left.i c_{r, 2}^{z}\right)$. Operator $\phi_{r}$ creates two fluxes in the plaquettes adjacent to the $z$ link in the elementary cell $r$. Note that $\phi_{r}^{+} \phi_{r}=\frac{1+u_{i j}}{2}$, and hence the ground-state wave function $|G\rangle$ satisfies $\phi_{r}^{+} \phi_{r}|G\rangle=|G\rangle$. The spin operator factorizes in the following way: $s_{r}^{z}=2 i \psi_{r}^{\alpha} \phi_{r}^{\alpha}$ where vectors $\psi_{r}^{\alpha}=$ $\left(\psi_{r}, \psi_{r}^{+}\right), \phi_{r}^{\alpha}=\left(\phi_{r}^{+}, \phi_{r}\right)$ are introduced and summation over $\alpha$ is implied. In the absence of a magnetic field, the correlation function of flux operators $\phi_{r}$ is local: $G_{\phi}(r, t)=\left\langle\phi_{r}^{+}(t) \phi_{0}(0)\right\rangle=\varphi(t) \delta_{r, 0}$, which leads to locality of the spin correlations. However, once the magnetic field is turned on, one finds $G_{\phi}(r, t)$ to be nonzero and proportional to $h_{z}^{2}$ at any $r$, which leads to spin correlation at large distances. We start from the expression for $\left\langle s_{r}^{z}(t) s_{0}^{z}(0)\right\rangle$, expanded up to the second order in $h_{z}$ :

$$
\begin{aligned}
\left\langle s_{r}^{z}(t) s_{0}^{z}(0)\right\rangle= & -\frac{h_{z}^{2}}{2} \sum_{r_{1}, r_{2}} \iint d \tau_{1} d \tau_{2} \\
& \times\left\langle T s_{r}^{z}(t) s_{0}^{z}(0) s_{r 1}^{z}\left(\tau_{1}\right) s_{r 2}^{z}\left(\tau_{2}\right)\right\rangle .
\end{aligned}
$$

The irreducible correlation function equals $g(t, \mathbf{r})=$ $\left\langle s_{r}^{z}(t) s_{0}^{z}(0)\right\rangle-\left\langle s_{0}^{z}(0)\right\rangle^{2}$, where

$$
\left\langle s_{0}^{z}(0)\right\rangle=-i h_{z} \sum_{r} \int d \tau\left\langle T s_{0}^{z}(0) s_{r}^{z}(\tau)\right\rangle .
$$

Thus we have to calculate two-spin and four-spin correlation functions. For these correlation functions to be nonzero, the flux configuration which results from the action of the two (four) spin operators on the ground state should coincide with the original flux configuration. In particular, the two-spin correlator vanishes unless $r=0$, so that we have $\left\langle T s_{0}^{z}(0) s_{r}^{z}(\tau)\right\rangle=u(\tau) \delta_{r, 0}$, with $u(\tau)=$ $\left\langle T s_{0}^{z}(0) s_{0}^{z}(\tau)\right\rangle$. It is clear that $u(-\tau)=u(\tau)$. The expression for the magnetization thus simplifies:

$$
\left\langle s^{z}\right\rangle=-2 i h_{z} \int_{0}^{\infty} u(\tau) d \tau
$$

Similarly,

$$
\begin{aligned}
\left\langle T s_{r}^{z}(t) s_{0}^{z}(0) s_{r_{1}}^{z}\left(\tau_{1}\right) s_{r_{2}}^{z}\left(\tau_{2}\right)\right\rangle= & f_{1}\left(r, t, \tau_{1}, \tau_{2}\right) \delta_{r 1, r} \delta_{r 2,0} \\
& +f_{2}\left(r, t, \tau_{1}, \tau_{2}\right) \delta_{r_{1}, 0} \delta_{r_{2}, r}
\end{aligned}
$$

where

$$
\begin{aligned}
& f_{1}\left(r, t, \tau_{1}, \tau_{2}\right)=\left\langle T s_{r}^{z}(t) s_{0}^{z}(0) s_{r}^{z}\left(\tau_{1}\right) s_{0}^{z}\left(\tau_{2}\right)\right\rangle, \\
& f_{2}\left(r, t, \tau_{1}, \tau_{2}\right)=\left\langle T s_{r}^{z}(t) s_{0}^{z}(0) s_{0}^{z}\left(\tau_{1}\right) s_{r}^{z}\left(\tau_{2}\right)\right\rangle .
\end{aligned}
$$

In the $t \rightarrow \infty$ limit, the leading contributions to $f_{1}$ and $f_{2}$ come from the regions $\tau_{1} \approx t, \tau_{2} \approx 0$ and $\tau_{1} \approx 0, \tau_{2} \approx t$, respectively. We will see that the product of spin operators at nearby times, e.g., $s_{r}^{z}(t) s_{r}^{z}\left(\tau_{2}\right)$ in the second case, reduces to the product of two fermion operators (up to some renormalization). It follows that the four-spin correlation function is asymptotically proportional to the density polarization function of free fermions.

Note that the Wick theorem is not directly applicable to spin averages because each spin operator creates both a fermion and some flux, the latter acting as a scattering potential for propagating fermions. To proceed with the calculation, one has to rewrite spin operators $s^{z}$ in Eq. (5) in terms of fermions $\psi$ and $\phi$, and then move the $\phi$ operators to the right, commuting them with exponential evolution factors. To this end, we use the identities

$$
\phi_{r} e^{i H t}=e^{i H_{r} t} \phi_{r}, \quad \phi_{r}^{\dagger} e^{i H_{r} t}=e^{i H t} \phi_{r}^{\dagger},
$$

where the Hamiltonian $H_{r}$ differs from the original Hamiltonian $H$ by inverting the sign of the $u$ variable which belongs to the $z$ link in the elementary cell $r: H_{r}=$ $H+V_{r}$, where $V_{r}=4 J\left(\psi_{r}^{+} \psi_{r}-\frac{1}{2}\right)$. In this way, all spin correlators can be represented as correlators of noninteracting fermions in the presence of external time-dependent potential. The calculation of $u(\tau)$ is a simple task discussed in Refs. [9,12]. Using the identity $e^{i H t} e^{-i H_{r} t}=$ $T \exp \left(-i \int_{0}^{t} V_{r}(\tau) d \tau\right)$, one arrives at the following result:

$$
u(\tau)=4\left\langle T \psi_{0}(\tau) \psi_{0}^{+}(0) e^{-i \int_{0}^{\tau} V_{0}\left(\tau^{\prime}\right) d \tau^{\prime}}\right\rangle \text { for } \tau>0 .
$$


The next step is to calculate $f_{1,2}\left(r, t, \tau_{1}, \tau_{2}\right)$. We consider explicitly all different time orderings in the expression (5); it is enough to choose $t>0$, since $g_{r}(-t)=g_{-r}^{*}(t)$ :
(1) $\tau_{2}>\tau_{1}>t>0$
(2) $\tau_{2}>t>0>\tau_{1}$
(3) $t>0>\tau_{2}>\tau_{1}$;
(4) $t>\tau_{2}>\tau_{1}>0$
(5) $t>\tau_{2}>0>\tau_{1}$
(6) $\tau_{2}>t>\tau_{1}>0$,

while the other 6 domains $1^{\prime}, \ldots, 6^{\prime}$ can be obtained by the permutation $\tau_{1} \leftrightarrow \tau_{2}$.

Let us illustrate how to perform the calculation of $f_{1,2}^{(j)}$ for the particular time domain $j=2$. We get for $f_{1}^{(2)}$ the following expression (summation over $\alpha, \ldots, \delta$ is implied):

$$
\begin{aligned}
f_{1}^{(2)} & =\left\langle s_{0}^{z}\left(\tau_{2}\right) s_{r}^{z}(t) s_{0}^{z}(0) s_{r}^{z}\left(\tau_{1}\right)\right\rangle=16\left\langle e^{i H \tau_{2}} \psi_{0}^{\alpha} \phi_{0}^{\alpha} e^{-i H \tau_{2}} e^{i H t} \psi_{r}^{\beta} \phi_{r}^{\beta} e^{-i H t} \psi_{0}^{\gamma} \phi_{0}^{\gamma} e^{i H \tau_{1}} \psi_{r}^{\delta} \phi_{r}^{\delta} e^{-i H \tau_{1}}\right\rangle \\
& =16\left\langle e^{i H \tau_{2}} \psi_{0} e^{-i H_{0} \tau_{2}} e^{i H_{0} t} \psi_{r} e^{-i H_{r, 0} t} \psi_{0}^{\dagger} e^{i H_{r} \tau_{1}} \psi_{r}^{\dagger} e^{-i H \tau_{1}} \phi_{0}^{\dagger} \phi_{r}^{\dagger} \phi_{0} \phi_{r}\right\rangle \\
& =-16\left\langle T \psi_{0}\left(\tau_{2}\right) \psi_{r}(t) \psi_{0}^{+}(0) \psi_{r}^{+}\left(\tau_{1}\right) e^{-i \int V_{1}^{(2)}(\tau) d \tau}\right\rangle .
\end{aligned}
$$

To proceed from the first to the second line, we used the fact that the only relevant sequence of superscripts is $\alpha \beta \gamma \delta=1122 \quad$ (recall that $\phi_{r}^{+} \phi_{r}|G\rangle=|G\rangle$ while $\phi_{r} \phi_{r}^{+}|G\rangle=0$ and $\left.\phi_{r}^{2}=\phi_{r}^{+2}=0\right)$. Similarly, for $f_{2}^{(2)}$ one obtains:

$$
f_{2}^{(2)}=16\left\langle T \psi_{r}\left(\tau_{2}\right) \psi_{r}^{+}(t) \psi_{0}(0) \psi_{0}^{+}\left(\tau_{1}\right) e^{-i \int V_{2}^{(2)}(\tau) d \tau}\right\rangle .
$$

The potentials $V_{1,2}^{(2)}(\tau)$ are piecewise-constant functions of time which can be easily read off the order of fermionic operators in (7) and (8):

\begin{tabular}{lcclcc} 
& $\left(-\infty ; \tau_{1}\right)$ & $\left(\tau_{1} ; 0\right)$ & $(0 ; t)$ & $\left(t ; \tau_{2}\right)$ & $\left(\tau_{2} ; \infty\right)$ \\
\hline$V_{1}^{(2)}(\tau)$ & 0 & $V_{r}$ & $V_{r}+V_{0}$ & $V_{0}$ & 0 \\
$V_{2}^{(2)}(\tau)$ & 0 & $V_{0}$ & 0 & $V_{r}$ & 0
\end{tabular}

In the same way exact expressions for $f_{i}^{(j)}$, analogous to (7) and (8), can be obtained for all other time domains $1, \ldots, 6$. However, they can hardly be evaluated exactly in the closed form. The problem of their calculation resembles the one encountered while exploring the Fermi edge singularity problem [13], so we can analyze it similarly.

The representation of spin correlation functions in the form (7) allows us to use the Wick theorem for fermions, which makes a diagrammatic expansion of $f_{i}^{(j)}$ over the potential $V_{i}^{(j)}$ possible. Note that apart from the normal Green function $G(t, \mathbf{r})=\left\langle T \psi(r, t) \psi^{+}(0,0)\right\rangle$, the anomalous Green function $F(t, \mathbf{r})=\langle T \psi(r, t) \psi(0,0)\rangle=$ $\left\langle T \psi^{+}(0,0) \psi^{+}(r, t)\right\rangle$ also has to be taken into account (we calculate both of them below). The sum of all diagrams for each of $f_{i}^{(j)}$ is of the form $f_{i}^{(j)}=16 e^{C_{i}^{(j)}} \cdot L_{i}^{(j)}$, where the first factor is the sum of closed-loop diagrams, and the second factor $L_{i}^{(j)}$ is the a sum of open-line diagrams.

The closed-loop contribution equals $e^{C_{i}^{(j)}\left(t, \tau_{1}, \tau_{2}\right)}=$ $\left\langle T e^{-i \int V_{i}^{(j)}(\tau) d \tau}\right\rangle$. In the limit of large time separation between pairs of points $\left\{t, \tau_{1}\right\},\left\{0, \tau_{2}\right\}$ or $\left\{t, \tau_{2}\right\},\left\{0, \tau_{1}\right\}$, the asymptotic form of $C_{i}^{(j)}$ can be simply determined. For example, the expressions for $C_{1,2}^{(2)}$ read:

$$
\begin{aligned}
& C_{1}^{(2)} \approx-i\left[\Omega\left(t+\tau_{2}-\tau_{1}\right)+\delta \Omega_{r} t\right], \\
& C_{2}^{(2)} \approx-i \Omega\left(\tau_{2}-\tau_{1}-t\right) .
\end{aligned}
$$

In this equation, $\Omega$ is the energy of the fermionic ground state in the presence of two adjacent fluxes $(\Omega \approx 0.04 \mathrm{~J}$, see [8]), while $\delta \Omega_{r}$ stands for the interaction energy of two flux pairs separated by distance $r$. Therefore, the factor $\exp \left[C_{i}^{(j)}\left(t, \tau_{1}, \tau_{2}\right)\right]$ rapidly oscillates with frequency $\Omega$.

Each term in the sum of open-line diagrams corresponds to a particular pairing of four fermionic operators in the product (7) or (8). For example, $L_{2}^{(2)}$ is given by the following equation:

$$
\begin{aligned}
L_{2}^{(2)}= & \left\langle T \psi_{r}\left(\tau_{2}\right) \psi_{r}^{+}(t)\right\rangle_{2}^{(2)}\left\langle T \psi_{0}(0) \psi_{0}^{+}\left(\tau_{1}\right)\right\rangle_{2}^{(2)} \\
& -\left\langle T \psi_{r}\left(\tau_{2}\right) \psi_{0}(0)\right\rangle_{2}^{(2)}\left\langle T \psi_{r}^{+}(t) \psi_{0}^{+}\left(\tau_{1}\right)\right\rangle_{2}^{(2)} \\
& -\left\langle T \psi_{r}\left(\tau_{2}\right) \psi_{0}^{+}\left(\tau_{1}\right)\right\rangle_{2}^{(2)}\left\langle T \psi_{0}(0) \psi_{r}^{+}(t)\right\rangle_{2}^{(2)},
\end{aligned}
$$

where $\langle T \cdots\rangle_{i}^{(j)}$ stands for $\left\langle T \cdots e^{-i \int V_{i}^{(j)} d \tau}\right\rangle e^{-C_{i}^{(j)}}$.

Finally, the spin correlation function $g(t, \mathbf{r})$ is given by the time integral over $\tau_{1}, \tau_{2}$ of the oscillating function $f_{i}^{(j)}$. The contribution from the domains $2+2^{\prime}$ reads:

$$
g_{i}^{(2)}(t, \mathbf{r})=-16 h^{2} \int_{-\infty}^{0} \int_{t}^{\infty} d \tau_{1} d \tau_{2} e^{C_{i}^{(2)}} L_{i}^{(2)} .
$$

Up to this point, all calculations have been exact for any $r, t$. To proceed further, we have to make some approximations. We use the inequality $t \gg J^{-1}$ and average over fast oscillations of $g_{i}^{(j)}(t, \mathbf{r})$ as a function of $t$. It is easy to see that $g_{2}^{(2)}$ has a slowly varying part because $V_{2}^{(2)}(\tau)=0$ for $\tau \in(0, t)$. On the other hand, $g_{1}^{(2)}$ is purely oscillating and vanishes upon the averaging. Considering the expression (10) for $g_{2}^{(2)}$, we find that the main contribution to it comes from $\tau_{1} \approx 0$ and $\tau_{2} \approx t$ (the result of integration is determined by a small neighborhood of the boundary points due to oscillations of the integrand), so the corresponding expression in (8) is of the form of fermionic density-density correlation function. Now we have to 
calculate $L_{2}^{(2)}$ for $\tau_{1} \approx 0$ and $\tau_{2} \approx t$. Note that for such time arguments, the external potential $V_{2}^{(2)}$ as a function of $\tau$ turns on for two short intervals (of the order of $\Omega^{-1}$ ), while the separation between the pulses is large, $t \gg J^{-1}$. In this case the long-time (or large-distance) asymptotics of the correlation function reads $\left\langle T \psi_{r}\left(\tau_{2}\right) \psi_{0}^{+}\left(\tau_{1}\right)\right\rangle_{2}^{(2)} \approx$ $G_{r}(t) \phi\left(0-\tau_{1}\right) \bar{\phi}\left(\tau_{2}-t\right)$, where $\phi(\tau), \bar{\phi}(\tau)$ are some dimensionless functions of $J \tau$. The double integral in (10) is thus factorized, and the renormalization due to the functions $\phi(t), \bar{\phi}(t)$ adds an overall numerical coefficient only, which we denote by $h_{0}^{-2}$ (it is the same for the contributions from all time domains). Calculating the dominant first term in (9) (the others oscillate as functions of $t$ ), we obtain $g_{2}^{(2)}$ :

$$
\begin{aligned}
g_{2}^{(2)}= & -16 h_{z}^{2}\left(\int_{\infty}^{0} u(\tau) d \tau\right) 2-16 h_{z}^{2} / h_{0}^{2}[F(t, \mathbf{r}) F(-t,-\mathbf{r}) \\
& +G(t, \mathbf{r}) G(-t,-\mathbf{r})] .
\end{aligned}
$$

Similar considerations are applicable for the other time domains $1, \ldots, 6$ show that all relevant contributions have a similar feature: the integration over $\left\{\tau_{1}, \tau_{2}\right\}$ is dominated by some neighborhood of points 0 and $t$. Collecting everything and subtracting $\left\langle s_{0}^{z}(0)\right\rangle^{2}$, we obtain

$$
g(t, \mathbf{r})=-64 \frac{h_{z}^{2}}{h_{0}^{2}}[F(t, \mathbf{r}) F(-t,-\mathbf{r})+G(t, \mathbf{r}) G(-t,-\mathbf{r})],
$$

where free fermion Green functions $G$ and $F$ are calculated below in Eq. (12). Thus we have found that the spin correlation function $g(t, \mathbf{r})$ is proportional to the densitydensity correlation function of band fermions, with the coefficient $\propto h_{z}^{2}$. The parameter $h_{0}$ in Eq. (11) can be estimated as $h_{0} \sim J$.

Now we turn to the calculation of fermionic Green functions $G(t, \mathbf{r})$ and $F(t, \mathbf{r})$ which enter (11). The expression for "vector" composed of these Green functions in the energy-coordinate representation reads:

$$
\begin{aligned}
& \left(G_{\epsilon}(\mathbf{r}), F_{\epsilon}(\mathbf{r})\right) \\
& \quad=\frac{2 i}{N} \sum_{\mathbf{p}} \frac{\left(\left(\epsilon+\operatorname{Im} f_{\mathbf{p}}\right) \cos (\mathbf{p r}),-\operatorname{Re} f_{\mathbf{p}} \sin (\mathbf{p r})\right)}{\epsilon^{2}-\left|f_{\mathbf{p}}\right|^{2}+i \delta},
\end{aligned}
$$

where $f(\mathbf{p})=2 i J\left(1+e^{i \mathbf{p} \mathbf{n}_{1}}+e^{i \mathbf{p} \mathbf{n}_{2}}\right)$ and $\mathbf{n}_{1,2}=\left( \pm \frac{1}{2}, \frac{\sqrt{3}}{2}\right)$ in the standard $(x, y)$ coordinates. We expand $f(\mathbf{p})$ near the conical point $\mathbf{K}=\left(\frac{2}{3} \pi, \frac{2}{\sqrt{3}} \pi\right)$ to get long-time behavior of $G(t, \mathbf{r})$, and $F(t, \mathbf{r})$. Substituting these asymptotics into Eq. (11), we obtain the final result:

$$
g(t, \mathbf{r})=\frac{16}{\pi^{2}}\left(\frac{h_{z}}{h_{0}}\right)^{2} \frac{\left(r^{2}-3(J t)^{2}\right) \cos ^{2}\left(\frac{2 \pi}{3} \mathbf{q}_{z}^{\perp} \mathbf{r}\right)-x^{2}}{\left(r^{2}-3(J t)^{2}\right)^{3}},
$$

where $\mathbf{q}_{z}^{\perp}=\hat{\mathbf{x}}$ is the unit vector along $x$, orthogonal to the direction of the $z-z$ link. The singularity of this expression at $r / t=\sqrt{3} J$ is cut off by the finite width of the Brillouin zone, which was sent to infinity while calculating integrals leading to Eq. (13). The anisotropy of correlation function (13) is due to our choice of magnetic field and spin correlations in the $z$ direction. Similar calculation for $x$ or $y$ components leads to analogous results with anisotropy vectors $\mathbf{q}_{x, y}^{\perp}$, which are perpendicular to the corresponding lattice links.

In conclusion, we have shown that, under weak perturbation due to a magnetic field, spin operators acquire a nonzero projection $\sim h^{2}$ on the density of band fermions; thus, long-range spin correlations appear. Therefore, the weakly perturbed honeycomb spin model may be considered as an example of a critical QSL. The same mechanism of coupling of spins to the fermion density can be realized for similar models on the decorated honeycomb lattice $[12,14]$. We expect the spin correlations to decay with a correlation length and time determined by the gap in the state of Ref. [14], whereas for the spin metal state [12], the asymptotic behavior $f(t, r) \propto[\max (t, r)]^{-2}$ is expected. Recent numerical results [10] demonstrate the appearance of longer spin correlations in the model [8] perturbed with an isotropic exchange term. An analytical study of this model would be of interest due to possible applications [11].

We are grateful to L. B. Ioffe and A. S. Ioselevich for useful discussions. This research was supported by the RFBR Grant No. 10-02-00554.

[1] P. W. Anderson, Mater. Res. Bull. 8, 153 (1973); P. Fazekas and P. W. Anderson, Philos. Mag. 30, 423 (1974).

[2] X. G. Wen, F. Wilczek, and A. Zee, Phys. Rev. B 39, 11413 (1989).

[3] X. G. Wen, Phys. Rev. B 44, 2664 (1991); , 65, 165113 (2002).

[4] R. Moessner and S. L. Sondhi, Phys. Rev. Lett. 86, 1881 (2001).

[5] C. Lhuillier, arXiv:cond-mat/0502464v1.

[6] G. Misguich, arXiv:0809.2257.

[7] M. Hermele, T. Senthil, and M. P. A. Fisher, Phys. Rev. B 72, 104404 (2005).

[8] A. Yu. Kitaev, Ann. Phys. (N.Y.) 321, 2 (2006).

[9] G. Baskaran, S. Mandal, and R. Shankar, Phys. Rev. Lett. 98, 247201 (2007).

[10] J. Chaloupka, G. Jackeli, and G. Khaliullin, Phys. Rev. Lett. 105, 027204 (2010).

[11] Y. Singh and P. Gegenwart, Phys. Rev. B 82, 064412 (2010).

[12] K. S. Tikhonov and M. V. Feigel'man, Phys. Rev. Lett. 105, 067207 (2010).

[13] P. Nozieres and C. de Dominicis, Phys. Rev. 178, 1097 (1969).

[14] H. Yao and S. A. Kivelson, Phys. Rev. Lett. 99, 247203 (2007). 